\title{
The role of basolateral amygdala orexin 1 receptors on the modulation of pain and psychosocial deficits in nitroglycerin-induced migraine model in adult male rats
}

\author{
Khadijeh Askari-Zahabi ${ }^{1}$, Mehdi Abbasnejad ${ }^{1}$, Razieh Kooshki ${ }^{2}$, Maryam Raoof ${ }^{3,4}$, Saeed Esmaeili-Mahani ${ }^{1}$, \\ Ali Mohammad Pourrahimi ${ }^{5}$, and Mahnaz Zamyad ${ }^{1}$
}

\author{
'Department of Biology, Faculty of Sciences, Shahid Bahonar University of Kerman, Kerman, Iran \\ ${ }^{2}$ Department of Biology, Faculty of Sciences, Lorestan University, Khorramabad, Iran \\ ${ }^{3}$ Department of Orofacial Pain and Dysfunction, Academic Centre for Dentistry Amsterdam (ACTA), University of Amsterdam and Vrije Universiteit \\ Amsterdam, Amsterdam, The Netherlands \\ ${ }^{4}$ Endodontology Research Center, Kerman University of Medical Sciences, Kerman, Iran \\ ${ }^{5}$ Kerman Neuroscience Research Center, Institute of Neuropharmacology, Kerman University of Medical Sciences, Kerman, Iran
}

Received December 21, 2020

Revised April 22, 2021

Accepted April 23, 2021

Handling Editor: Sang Hun Kim

\section{Correspondence}

Ali Mohammad Pourrahimi

Kerman Neuroscience Research Center, Institute of Neuropharmacology, Jehad Blvd, Ebn Sina Avenue, Kerman 7619813159, Iran

Tel: +983432264196

Fax: +9832264198

E-mail: a1poorrahimi2001@yahoo.com
Background: Migraine headaches have been associated with sensory hyperactivity and anomalies in social/emotional responses. The main objective of this study was to evaluate the potential involvement of orexin 1 receptors (Orx1R) within the basolateral amygdala (BLA) in the modulation of pain and psychosocial dysfunction in a nitroglycerin (NTG)-induced rat model of migraine.

Methods: Adult male Wistar rats were injected with NTG $(5 \mathrm{mg} / \mathrm{kg}$, intraperitoneal) every second day over nine days to induce migraine. The experiments were done in the following six groups (6 rats per group): untreated control, NTG, NTG plus vehicle, and NTG groups that were post-treated with intra-BLA microinjection of Orx1R antagonist SB-334867 (10, 20, and $40 \mathrm{nM})$. Thermal hyperalgesia was assessed using the hot plate and tail-flick tests. Moreover, the elevated plus maze (EPM) and open field (OF) tests were used to assess anxiety-like behaviors. The animals' sociability was evaluated using the three-chamber social task. The NTG-induced photophobia was assessed using a light-dark box.

Results: We observed no change in NTG-induced thermal hyperalgesia following administration of SB-334867 (10, 20, and $40 \mathrm{nM})$. However, SB-334867 (20 and $40 \mathrm{nM}$ ) aggravated the NTG-induced anxiogenic responses in both the EPM and OF tasks. The NTG-induced social impairment was overpowered by SB-334867 at all doses. Time spent in the dark chamber of light-dark box was significantly increased in rats treated with SB-334867 (20 and $40 \mathrm{nM} / \mathrm{rat}$ ).

Conclusions: The findings suggest a role for Orx1R within the BLA in control comorbid affective complaints with migraine in rats.

Key Words: Amygdala; Anxiety; Basolateral Nuclear Complex; Hyperalgesia; Migraine Disorders; Nitroglycerin; Orexins; Orexin Receptors; Orexin Receptor Antagonists; Rats. (c) This is an open-access article distributed under the terms of the Creative Commons Attribution Non-Commercial License (http://creativecommons.org/licenses/by-nc/4.0/), which permits unrestricted non-commercial use, distribution, and reproduction in any medium, provided the original work is properly cited.

(c) The Korean Pain Society, 2022
Author contributions: Khadijeh Askari-Zahabi: Investigation; Mehdi Abbasnejad: Supervision; Razieh Kooshki: Project administration; Maryam Raoof: Writing/manuscript preparation; Saeed Esmaeili-Mahani: Formal analysis; Ali Mohammad Pourrahimi: Funding acquisition; Mahnaz Zamyad: Investigation. 


\section{INTRODUCTION}

Migraine is a disabling headache disorder that causes both emotional and sensory disturbances [1]. The comorbidity between migraine and psychiatric disorders is frequently indicated. In particular, migraine sufferers exhibited higher levels of anxiety and stress as well as lower sociability than the healthy individuals [2]. Considering the strong link between the psychosocial and physical aspects of migraine, the improvement of knowledge on potential therapeutic targets is significant in the treatment of this debilitating headache.

The neural mechanism(s) underlying the pathogenesis of migraine is extremely complex and still needs to be addressed in research. In addition to multiple brainstem pain-modulation circuits, a dysfunctional neurolimbic pain network can play a crucial role in the triggering and progression of migraine [3]. There is growing evidence that the amygdala, and more specifically its central (CeA) and basolateral (BLA) areas, may reflect an important part of migraine pathophysiology [4]. Moreover, altered functional connectivity between the amygdala nuclei and visceroceptive cortex has been reported in migraine [4]. A human study found increased functional connectivity of the left amygdala and a decreased level of the right amygdala activity in chronic compared to episodic migraine [5].

Because of its highly lipophilic structure nitroglycerin (NTG) easily passes the blood-brain barrier and lead to nitric oxide production and vasodilation [6]. NTG-induced migraine has been presented as a reliable experimental model of migraine in humans. Oral NTG administration induced migraine-like headache and decreased head pain threshold in humans [7].

Orexin-A is also known as hypocretin-1, is a neuropeptide that is synthesized by a small number of hypothalamic neurons. Upon its binding to the orexin 1 receptor (OrxlR), target cell activation occurs [8]. Due to the projections to the brainstem and limbic regions of the pain matrix, including the amygdala subregions, the orexin neurons are not only involved in sensory processing, but also emotional control of nociceptive reactions. The blockade of OrxlR in the BLA has been shown to induce facilitation of fear extinction consolidation through the activation of BLA outputs to the infralimbic medial prefrontal cortex [9]. Interestingly, orexin connectivity with melanin-concentrating hormone receptor systems in the BLA has been implicated in regulating sociability and mood-related behaviors [10].

The outcomes of previous researches identify an association between migraine and Orx1R [11]. It has been indicated that the activation of OrxlR suppresses trigeminal neural firing induced by dural electrical stimulation in rats [11]. The involvement of the BLA circuitries in migraine headache, and their related emotional disorders, has been supported by different studies. However, little is known about the role of OrxlR expression in the BLA in this regard; therefore, we investigated whether OrxlR signaling in the BLA modulates NTG-induced anxiety and social interaction deficits in rats.

\section{MATERIALS AND METHODS}

\section{Animals}

Adult male Wistar rats (230-260 g body weight) were used in this study. Because of the difficulties in controlling female endocrine balance, only male rats were studied. The rats were housed in a temperature-controlled room $\left(23^{\circ} \mathrm{C} \pm 1^{\circ} \mathrm{C}\right)$ under a regular 12-hour light/dark cycle with ad libitum access to food and water. All experimental procedures were reviewed and approved by the Ethics Committee of Kerman University of Medical Sciences (IR.KMU. REC1399.267).

\section{Drugs}

NTG was purchased from Caspian Tamin Pharmaceutical Company (Rasht, Iran). The OrxlR antagonist SB-334867 (Sigma-Aldrich, St. Louis, MO) was dissolved in dimethylsulfoxide (DMSO) and then diluted into artificial cerebrospinal fluid (CSF). The final concentration of DMSO was $0.1 \%$.

\section{Surgical procedure}

The rats were anesthetized with a ketamine $(65 \mathrm{mg} / \mathrm{kg} /$ intraperitoneal [i.p.]) and xylazine (10 mg/kg/i.p.) mixture, and fixed on a stereotaxic frame (Stoelting, Wood Dale, IL). A 23-gauge stainless steel guide cannula was implanted bilaterally into the BLA at the following coordinates: 7.8 $\mathrm{mm}$ posterior to the bregma, $0.6 \mathrm{~mm}$ lateral to the midline, and $5.9 \mathrm{~mm}$ ventral to the cortical surface [12]. The animals recovered from the surgical procedure for 1 week before the experiments. At the end of the experiments, using a bilateral injection of $0.5 \mu \mathrm{L}$ of methylene blue through the cannula, all rats were anatomically inspected for the cannula's position. Rats with misplaced cannulas were excluded from the analysis and replaced by correct ones. Fig. 1A-C shows the surgical procedure. Fig. 1D shows a typical section of the BLA taken from the Paxinos and Watson rat brain atlas and a representative photograph exhibiting appropriate cannula implantation into the BLA region. 

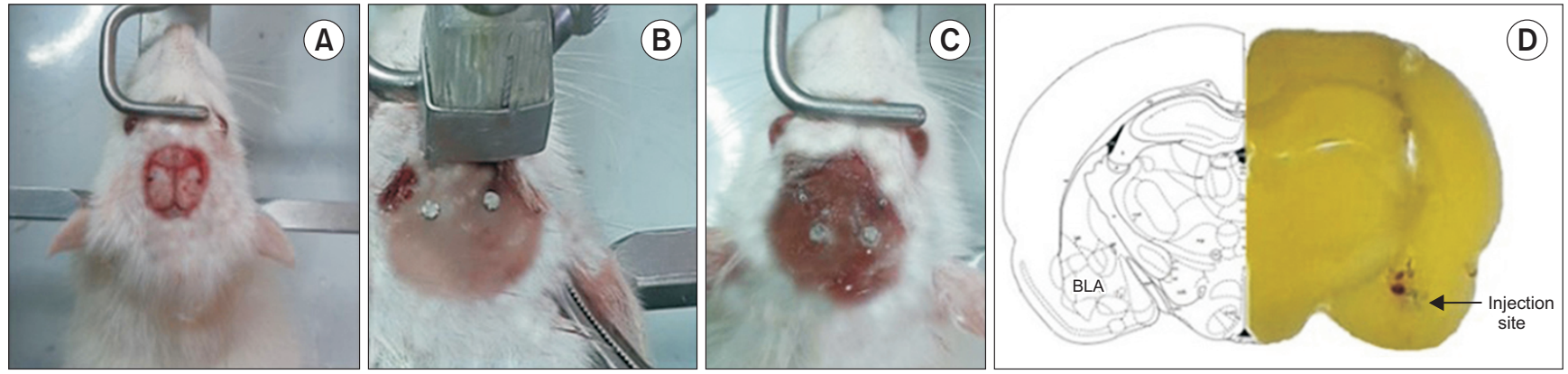

Fig. 1. Stereotaxic surgical procedure for guide cannula implantation in the basolateral amygdala (BLA) (A-C), a typical section of BLA taken from the Paxinos and Watson rat brain atlas and a representative photographs exhibiting appropriate cannula implantation into the BLA region (D).

\section{Microinjection}

The drugs were delivered into the BLA through a 30-gauge stainless steel cannula attached to a $1 \mu \mathrm{L}$ Hamilton syringe via polyethylene catheter tubing. The tip of the injector protruded $1 \mathrm{~mm}$ below the tip of the guide cannula. Each animal received bilateral injections of $1 \mu \mathrm{L}(1 \mu \mathrm{L} / \mathrm{min}$ per side).

\section{Experiment groups}

In total, 72 rats were used. The rats were randomly divided into 12 groups (6 rats in each group). The simple random sampling method was used. The experimental procures, groups used in each section, and number and the order of behavioral tests are descripted in the flow diagram (Fig. 2). The sample size and number of animal per groups were designated according to the pilot experiments and previous studies in the field in our lab $[13,14]$.

\section{Assessment of thermal sensitivity}

\section{1) Tail flick test}

The tail-flick test was used to assess NTG-induced changes in thermal pain sensitivity. The rats were gently restrained, and radiant heat was applied to the base of the tail. The time taken to withdraw from the heat stimulus was recorded as the withdrawal latency. Tail-flick latency was verified for each rat three times at two-minute intervals, and the average time was recorded. A cutoff time was established at 12 seconds to prevent tissue injury.

\section{2) Hot plate test}

The hot plate test was used to measure thermal hyperalgesia. Rats were placed individually on a metal surface maintained at a constant temperature $\left(52^{\circ} \mathrm{C} \pm 2^{\circ} \mathrm{C}\right)$, and latencies for licking, lifting, or jumping responses were re-

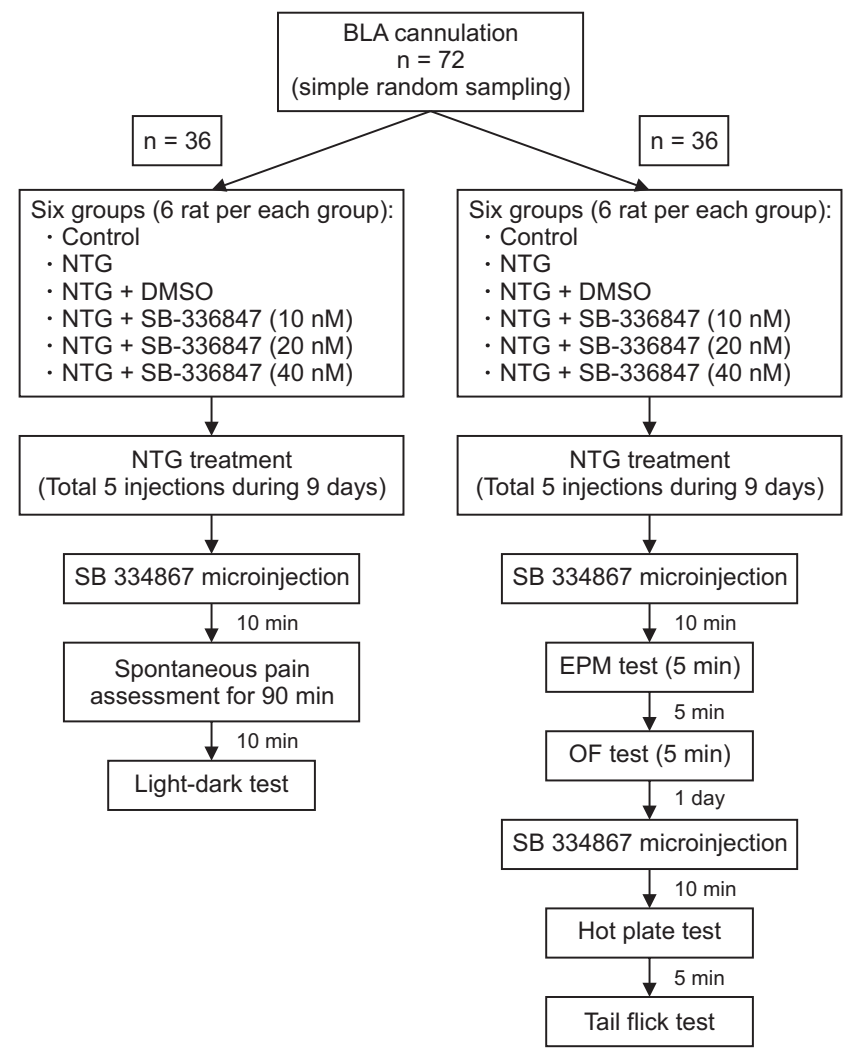

Fig. 2. The flow chart of study recruitment. BLA: basolateral amygdala, NTG: nitroglycerin, DMSO: dimethylsulfoxide, EPM: elevated plus maze, OF: open field.

corded. To prevent tissue damage, a 14 seconds cutoff was used.

\section{Assessment of anxiety-like behaviors}

\section{1) Elevated plus maze (EPM) test}

The maze was $50 \mathrm{~cm}$ above the floor and consisted of two open arms that were surrounded by $0.5-\mathrm{cm}$ high transparent Plexiglas ledges and two closed arms of the same size $(60 \mathrm{~cm})$ that were enclosed by $40-\mathrm{cm}$ high wooden walls. 
The four arms met in a $5 \mathrm{~cm}$ center square region. Each rat was placed at the junction of the four arms, facing an open arm, and allowed to freely explore the maze for 5 minutes. The number of open arm entries and time spent in open and closed arms were recorded via a video tracking system.

\section{2) Open field (OF) test}

The apparatus was constructed of a black-painted wooden box $(70 \times 70 \mathrm{~cm})$ surrounded by $30 \mathrm{~cm}$ high walls. Each rat was placed in the center of the box and allowed to explore for 5 minutes freely. Time spent, and the number of entries into the central zone (covering $40 \times 40 \mathrm{~cm}$ ) were considered in the analysis of anxiety-related behavior.

\section{Three-chambered social approach task}

The maze consisted of three equal-sized rectangular Plexiglas compartments $(20 \times 40 \times 47 \mathrm{~cm})$, with small openings $(5 \times 3 \mathrm{~cm})$ in the dividing walls that allow access into each chamber. The compartments could be closed with removable slide doors. Two dome-shaped wire cages $(9 \mathrm{~cm}$ in diameter, $11 \mathrm{~cm}$ in height, with vertical rods spaced $0.5 \mathrm{~cm}$ apart) were set in the lateral compartments. A three-step procedure including, habitation, sociability, and social discrimination, was conducted. In the habituation session, each rat was placed in the middle box while the slide doors were open, and allowed to move freely through all three chambers for 5 minutes. In the sociability step, a novel stranger male rat (stranger 1) was confined in one of the wire cages located in the corner of each lateral partition. The wire cup prevented the animal from coming into direct contact and fighting with the other rat. The subject rat was placed in the middle part, with removed doors, and allowed to explore all three chambers for 10 minutes. In the social discrimination trial, a second unfamiliar rat (stranger 2) was placed in the empty dome-shaped wire cage in the opposite compartment. The rat was then allowed to explore the apparatus for 10 minutes with the doors opened. The total time spent in each chamber and the time spent around each wire cage during the two 10 minutes sessions were measured. Besides, a discrimination index (DI) was calculated as follows:

$\mathrm{DI}=$ (Time spent near the cage of stranger $1 /$ Time spent near the cage of stranger $1+$ stranger 2$) \times 100 \%$

\section{Assessment of NTG-induced spontaneous pain behavior}

These behaviors were included: the time of continuous shaking of the head, ipsilateral hind paw facial grooming, and freezing (immobility) behavior characterized by all four paws in contact with the floor without any movement of the vibrissae [15].

\section{Light aversion test}

The maze consists of a chamber divided into a dark and a light compartment. Each rat was placed in the light chamber first and the behavior of the rat was evaluated over a 10 minutes period. The total time spent in the light and dark compartments was measured to qualify photophobia behavior in NTG-induced migraineurs rats.

\section{Statistical analysis}

All values are presented as the mean \pm standard error of the mean. Data related to migraine-like spontaneous pain behaviors, the hot plate, tail-flick, OF, EPM, and light-dark tests were analyzed with parametric one-way analysis of variance, followed by Tukey's post-hoc test. However, the data of the three-chambered social approach task was analyzed by the non-parametric Kruskal-Wallis test. Moreover, the Mann-Whitney $U$-test was performed to further examine differences between experimental groups. $P$ values of less than 0.05 were regarded as statistically significant.

\section{RESULTS}

\section{Thermal hypersensitivity}

1) Hot plate test

There were significant differences in hot plate latencies among the studied groups $\left[F_{(5,35)}=12.413, P<0.001\right]$. As shown in Fig. 3A, NTG significantly decreased the hot plate reaction time $(2.275 \pm 0.594)$ as compared to the control group $(5.243 \pm 0.592)(P<0.001)$. However, intra-BLA administration of SB-334867 at the doses of 10, 20, and 40 nM did not change NTG-induced increased thermal sensitivity in rats (Fig. 3A).

\section{2) Tail flick test}

The findings proved significant differences in thermal latency scores in the tail-flick test among the studied groups $\left[F_{(5,35)}=5.573, P<0.001\right]$. Treatment with NTG $(7.085 \pm 0.565)$ significantly shortened tail-flick latency in comparison to that of the control rats $(11.07 \pm 0.518)(P<0.001)$. However, post-treatment with OrxlR antagonist SB-334867 (10, 20, and $40 \mathrm{nM}$ ), did not affect the thermal sensitivity in NTG- 

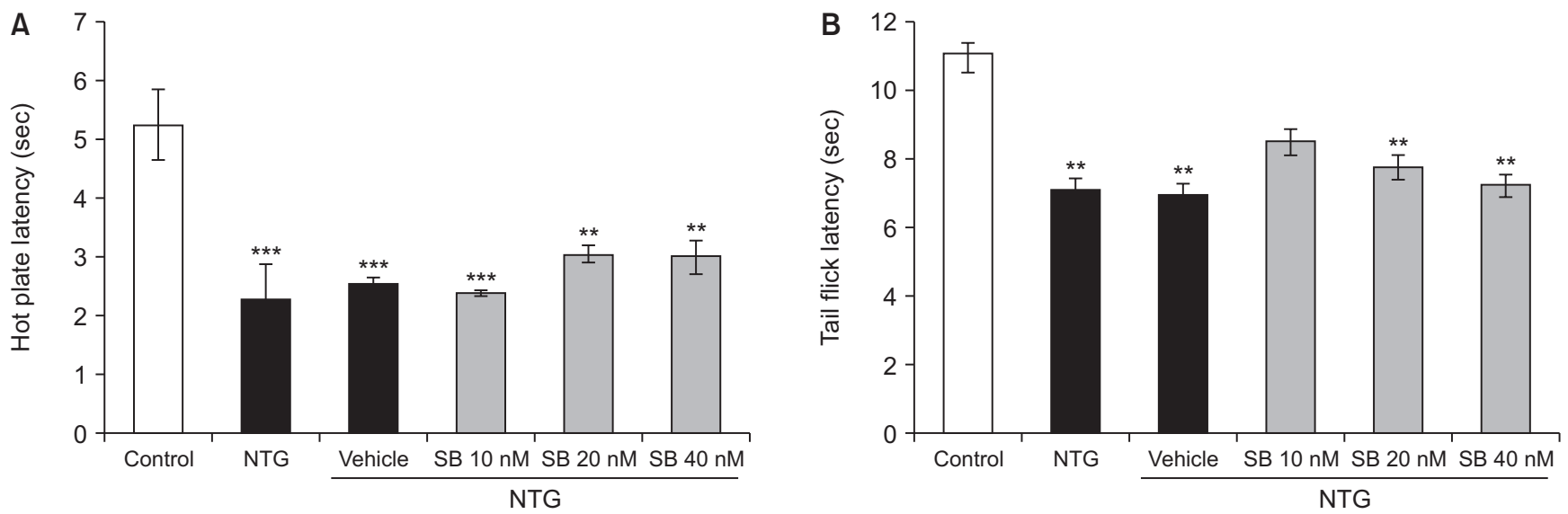

Fig. 3. The hot plate (A) and tail flick (B) latencies among the different experimental groups ( $n=6$ per group). Graphs represent the mean \pm standard error of the mean. NTG: nitroglycerin, SB: SB-334867. $* * P<0.01$ vs. control group, $* * * P<0.001$.

Table 1. Evaluation NTG-induced spontaneous migraine-like headache symptoms between groups

\begin{tabular}{|c|c|c|c|}
\hline \multirow{2}{*}{ Group } & \multicolumn{3}{|c|}{ Spontaneous orofacial pain behavior } \\
\hline & Head shaking & Ipsilateral hind paw facial grooming & Freezing \\
\hline Intact & $230.83 \pm 32.84$ & $10.00 \pm 5.16$ & $768.33 \pm 39.61$ \\
\hline NTG & $467.50 \pm 53.45 * *$ & $39.16 \pm 12.93$ & $1,958.33 \pm 204.53 * * *$ \\
\hline NTG + DMSO & $379.16 \pm 24.67$ & $42.50 \pm 8.73$ & $1,729.00 \pm 107.87 * * *$ \\
\hline $\mathrm{NTG}+\mathrm{SB}(10 \mathrm{nM})$ & $467.50 \pm 49.87 * *$ & $92.50 \pm 9.81 * * *$ & $4,240.00 \pm 65.01 * * *$,\#\#\# \\
\hline $\mathrm{NTG}+\mathrm{SB}(20 \mathrm{nM})$ & $594.16 \pm 49.60 * *$ & $93.33 \pm 10.46 * * *, \#$ & $3,778.33 \pm 96.89 * * *$ \\
\hline $\mathrm{NTG}+\mathrm{SB}(40 \mathrm{nM})$ & $770.83 \pm 31.07 * * *$,\#\# & $110.00 \pm 20.08 * * *, \# \#$ & 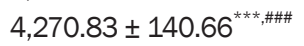 \\
\hline
\end{tabular}

Values are presented as mean \pm standard error of the mean $(n=6)$.

NTG: nitroglycerin, DMSO: dimethylsulfoxide, SB: SB-334867.

${ }^{* *} P<0.01, * * * P<0.001$ vs. control group, ${ }^{\#} P<0.05,{ }^{\# \#} P<0.01,{ }^{\# \#} P<0.001$ vs. NTG group.

treated rats (Fig. 3B).

\section{Evaluation of NTG-induced spontaneous migraine-like pain}

There were significant differences between groups in head shaking, ipsilateral hind paw facial grooming and freezing behaviors. As shown in Table 1, the time of the head shaking and freezing behavior was significantly increased in rats treated by NTG $(P<0.001)$. However, the time course of ipsilateral hind paw facial grooming behaviors did not show significant change in rats treated with NTG or NTG plus vehicle as compared to the control group. NTGinduced freezing behavior was exaggerated in rats treated with SB-334867 (40 nM/rat) as compared to the NTG group. Moreover, ipsilateral hind paw facial grooming was significantly increased in rats treated with SB-334867 at $20(P=0.038)$ and $40 \mathrm{nM} /$ rat $(P=0.003)$ before administration of NTG as compared to the control group. In addition, NTG-induced freezing behavior was significantly increased in rats treated with intra-BLA administration of SB-334867 at all doses $(10,20$, and $40 \mathrm{nM} / \mathrm{rat})(P<0.001)$ (Table 1).

\section{Anxiety-like behaviors}

1) EPM test

The groups showed significant differences in the time spent $\left[F_{(5,35)}=20.065, P<0.001\right]$ and the frequency of visits into the open arms $\left[F_{(5,35)}=23.023, P<0.001\right]$. As shown in Fig. 4A, the time spent in the open arms was significantly decreased in rats treated with NTG $(56.95 \pm 11.950)$ in comparison with the control group $(133.8 \pm 13.657)(P<0.001)$. Intra-BLA administration of SB-334867 at the dose of 20 $\mathrm{nM}(15.166 \pm 7.77)$ decreased the time spent in open arms in NTG-treated rats $(P=0.004)$. Furthermore, administration of NTG significantly decreased the number of entries into the open arms $(8.833 \pm 1.40)$ as compared to nontreated control rats $(16.833 \pm 1.55)(P<0.001)$. The effects of NTG were exaggerated by intra-BLA administration of SB-334867 at the doses of $40 \mathrm{nM}(P<0.001)$ and $20 \mathrm{nM}(P=$ 

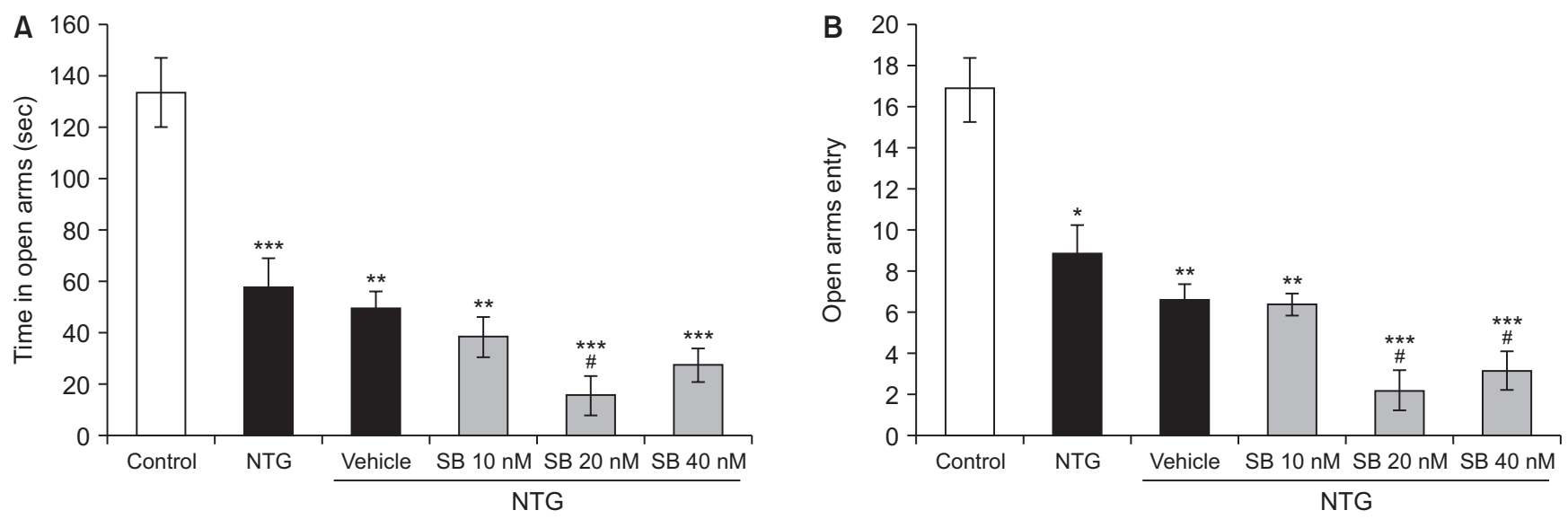

Fig. 4. The time spent (A) and the number of entries (B) into the open arms of the elevated plus-maze during a 5 minutes test in nitroglycerin (NTG)-treated rats and NTG groups received intra-basolateral amygdala infusion of SB-334867 (10,20, and $40 \mathrm{nM} / \mathrm{rat})(\mathrm{n}=6$ per group). Graphs represent the mean \pm standard error of the mean. ${ }^{*} P<0.05, * * P<0.01, * * * P<0.001$ vs. control group, ${ }^{\sharp} P<0.05$ vs. NTG.
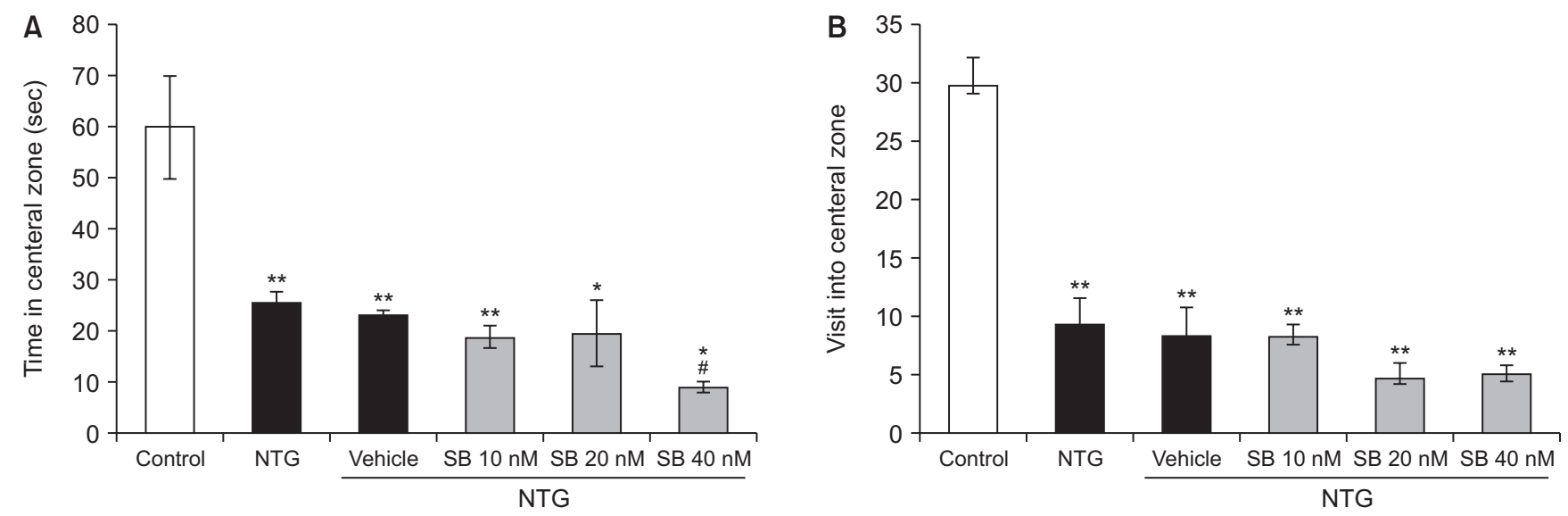

Fig. 5. The time spent (A) and the number of entries (B) in the central zone of the open filed during a 5 minutes test in nitroglycerin (NTG)-treated rats and NTG groups that received intra-basolateral amygdala infusion of SB-334867 (10,20, and $40 \mathrm{nM} / \mathrm{rat}) . \mathrm{n}=6$ per experimental group. Graphs represent the mean \pm standard error of the mean. ${ }^{*} P<0.05, * * P<0.01$ vs. control group, ${ }^{\#} P<0.05$ vs. NTG.

0.002) (Fig. 4B).

\section{2) OF test}

The groups showed significant differences in the time spent $\left[F_{(5,35)}=11.425, P<0.001\right]$ and the frequency of entries into the central zone $\left[F_{(5,35)}=24.162, P<0.001\right]$. As shown in Fig. 5A, rats treated with NTG $(25.283 \pm 2.436)$ spent significantly less time in the central zone of the maze as compared to the control group $(59.9 \pm 10.302)(P<0.001)$. Moreover, the time spent in the central zone was significantly decreased in NTG rats that received intra-BLA administration of SB-334867 at the dose of $40 \mathrm{nM}(8.816 \pm$ 1.079) $(P=0.033)$. In addition, NTG significantly decreased the number of entries into the central zone of the maze $(9.166 \pm 2.344)$. However, microinjections of SB-334867, at the different doses, did not alter NTG-related decreased entries into the central zone of the maze (Fig. 5B).

\section{Light aversion test}

A light-dark box was used to assess the NTG-induced photophobia behavior of the rats. The hotophobia behavior was significantly different between the groups $\left[F_{(5,35)}=\right.$ 36.320, $P<0.001]$. As shown in Fig. 6 , time spent in the dark chamber was significantly increased in rats treated with NTG or NTG + DMSO as compared to control group $(P<$ 0.001). Intra-BLA administration of SB-334867 (20 and 40 $\mathrm{nM} / \mathrm{rat}$ ) significantly increased NTG-induced increases in time spent in the dark chamber of the box $(P<0.001)$.

\section{Social interaction task}

In the sociability test, as shown in Fig. $\mathbf{7 A}$, the time that NTG rats spent in the compartment with an unfamiliar rat (stranger 1) was significantly decreased compared to that of the control group $(P=0.015)$. Moreover, intra-BLA 
infusion of SB-334867 (20 and $40 \mathrm{nM} / \mathrm{rat}$ ) significantly increased the effects of NTG $(P<0.001)$. Besides, the NTG group and NTG groups post-treated with SB-334867 (20

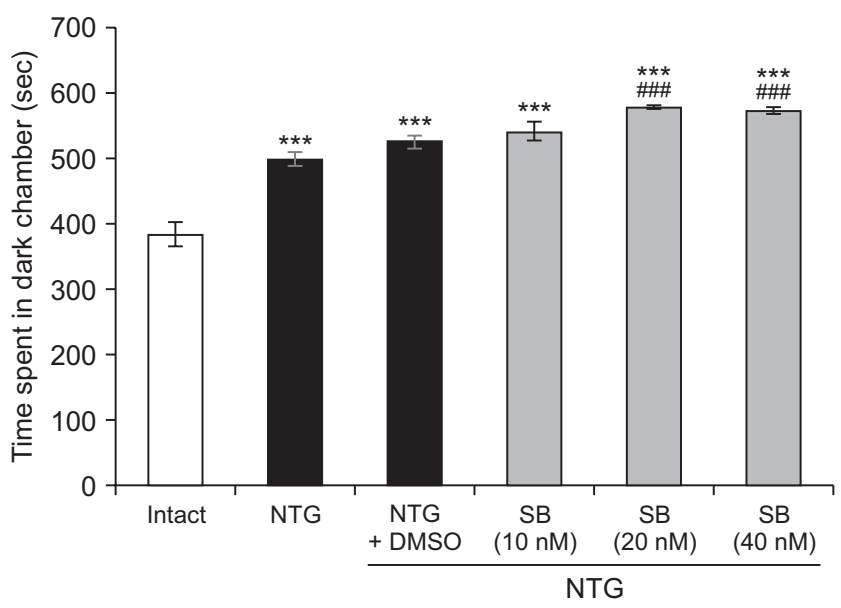

Fig. 6. Assessment of light aversion behavior in the light dark box test. The data in each graph are represented as mean \pm standard error of the mean values $(n=6)$. NTG: nitroglycerin, DMSO: dimethylsulfoxide, SB:

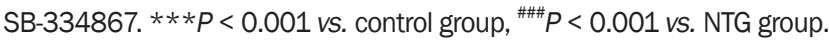
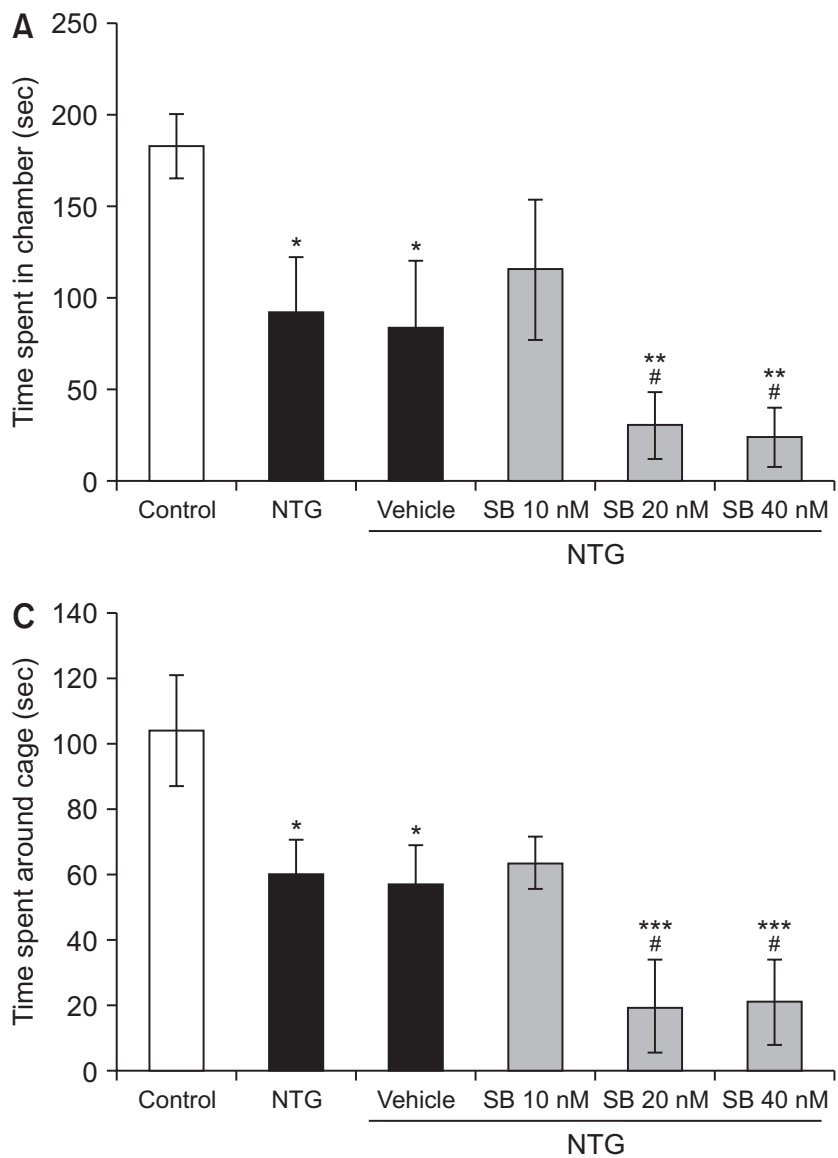

and $40 \mathrm{nM} / \mathrm{rat}$ ) spent a significantly shorter time around the wire cage containing stranger 1 than that of the control rats $(P<0.001)($ Fig. $7 \mathrm{C})$. The social DI and the time that NTG treated rats spent in the chamber containing stranger 2 were significantly decreased compared to the intact animals $(P=0.026)$. The NTG effects were strengthened significantly following intra-BLA administration of SB-334867 at $10(P=0.041) 20(P=0.009)$ and $40 \mathrm{nM} / \mathrm{rat}(P=$ 0.004) (Fig. 7B, D).

\section{DISCUSSION}

In the present study, we showed that the inactivation of OrxlR in the BLA of rats suffering NTG- induced migraine increased photophobia, anxiety-like behavior, and social interaction deficits. In addition, OrxlR antagonism was associated with increases in spontaneous migraine-like headache behaviors in NTG-treated rats. Besides, NTG increased thermal pain sensitivity in both the tail-flick and hot plate tests. However, pharmacological blockade of the OrxlR did not affect the NTG-induced thermal hyperalge-
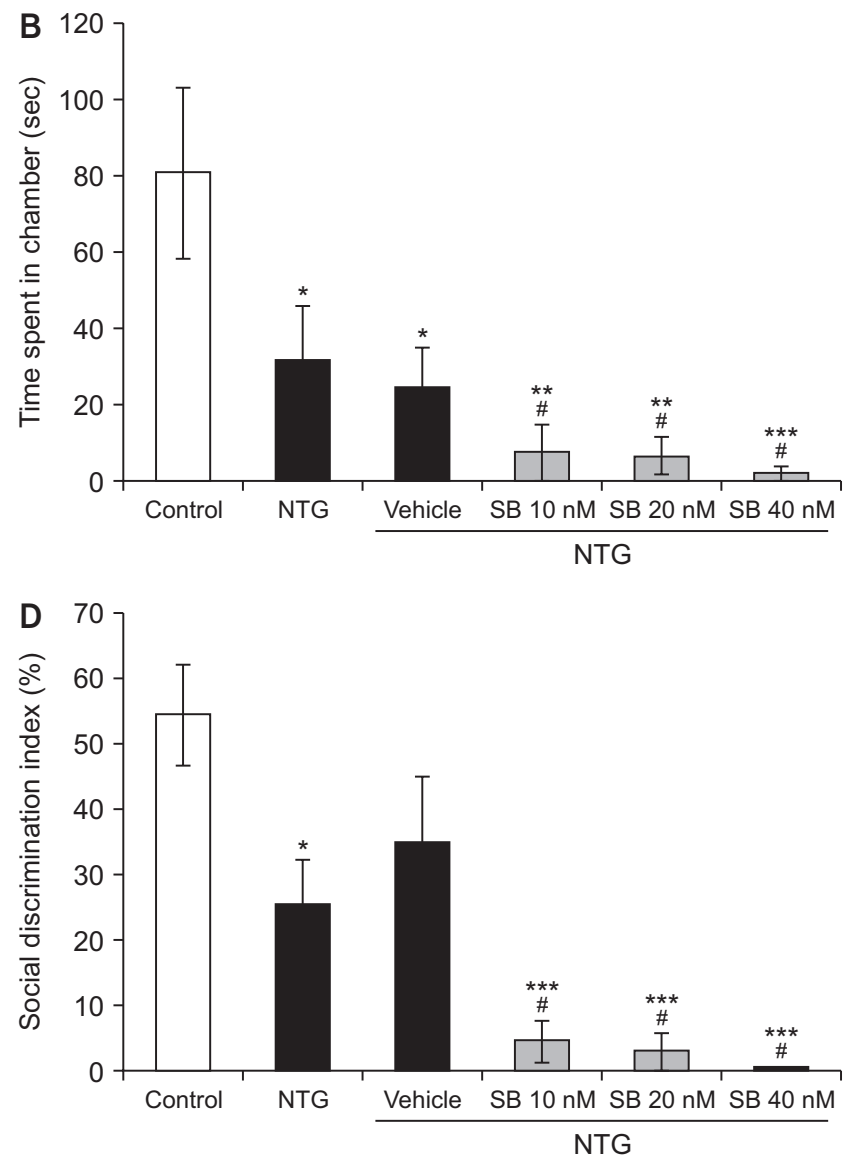

Fig. 7. Sociability in the three-chambered task. The time spent in the chamber containing stranger 1 (A) and stranger 2 (B), and the time spent in close proximity with the cage containing stranger 1 (C). Social discrimination (D). $n=6$ per experimental group. Bar graphs represent mean \pm standard error of the mean. NTG: nitroglycerin, SB: SB-334867. ${ }^{*} P<0.05, * * P<0.01$, and $* * * P<0.001$ vs. control, ${ }^{\#} P<0.05$ NTG and NTG plus vehicle. 
sia.

Previous data strongly suggested that NTG evokes sensory hypersensitivity associated with migraine $[16,17]$. Chronic administration of NTG causes neuronal hyperactivity in the trigeminal and midbrain nociceptive pathways [18], which can induce central sensitization by over-expression of various pain signaling mediators [19]. Suppression of NTG-evoked pain sensitivity in rodents given anti-migraine medicines provides support for the validity of the NTG-induced model of migraine [20].

Somatosensory hypersensitivity and reduced cutaneous pain thresholds have been distinguished as clinical symptoms associated with migraine headache [21]. The majority of migraineurs have been shown physiologic evidence for thermal and mechanical cutaneous stimuli during the headache $[22,23]$. For example, it has been indicated that interictal cutaneous pain thresholds are lower in episodic and chronic migraine patients compared to non-migraine controls [24]. Specially, NTG-induced migraine has been associated with a significant heat pain threshold reduction in humans [7]. So, here, thermal hyperalgesia was evaluated as a relevant indication for migraine.

The data showed that OrxlR antagonism in the BLA enhanced NTG-induced spontaneous orofacial pain. In specific regard to NTG-related migraine pain, activation of OrxlR in the ventrolateral preaqudactal gray matter has increased spontaneous pain behaviors in rats. In a clinical study, patients with episodic migraine showed an increase in orexin-A concentration as measured in the CSF. The hypothalamic orexinergic neurons are densely projected to other brain systems involved in migraine pathophysiology, especially limbic structures and the trigeminal cervical complex of the brainstem, implicated in the transmission of the trigeminal nociceptive to higher thalamic and cortical areas. It shows the importance of orexinergic systems to control attack susceptibility and modulation of affective and emotional symptoms relevant to migraine.

Following the blockade of Orx1R in the BLA, NTGinduced anxiety-like behavior was overstated in both the EPM and OF tasks. This finding suggests the role of endogenous orexin signaling within the BLA in modulating anxiety in migraine. Also, dysfunction of the orexinergic system has been shown to be related to pathological anxiety states [25]. Interestingly, abnormally elevated levels of orexin have been found in the cerebrospinal fluid of patients with migraine or panic disorder $[26,27]$. It has also been indicated that intra-amygdala infusion of SB-334867 enhances fear extinction associated with increased neuronal activity in the BLA and infralimbic cortex [28].

Although the mechanism(s) by which the Orx1R system within the BLA modulates NTG-induced anxiety is not entirely clear, it appears to act by receptor activation and subsequent alterations in intracellular targets. The OrxlR activation evokes a wide range of intracellular signaling cascades, including cAMP-dependent protein kinase $\mathrm{A}$ (cAMP-PKA) and the extracellular signal-regulated kinase/mitogen-activated protein kinase (ERK/MAPK) [29]. The crucial roles of such pathways in controlling anxiety and affective responsiveness have been suggested in previous studies [30,31].

Intra-BLA administration of PKA agonist decreased the anxiogenic, social defeat stress-induced behaviors in both the EPM and OF tests. Moreover, the antidepressant clomipramine reduced anxiety and up-regulated PKA levels in the BLA [32]. It has been indicated that the activation of the ERK/MAPK signaling pathway is necessary for the acquisition of conditioned fear [33], whereas, the activation of ERK2 in the BLA increased anxiety-like behaviors in stressed rats [34]. Altogether, these results suggest the potential dual effects (excitatory or inhibitory) of the orexin system and its downstream targets on anxiety disorders. So, it is not possible to assume that hyperactivity of the orexin system in the BLA acts as functional compensation to preserve a normal physiological state through the masking of anxiogenic-like effects induced by NTG. Further studies may then be needed to identify the interventional effects of OrxlR signaling on migraine-related anxiety.

We also found that bilateral inhibition of OrxlR in the BLA enhanced social interaction dysfunction in NTGtreated rats. In line with our observation, Abbas et al. [35], also indicated impaired sociability and social novelty preferences in OrxlR knockout mice. Moreover, selective ablation of orexin neurons in orexin/ataxin-3 transgenic mice has been associated with deficits in long-term social memory. Such effects were suppressed by intra-nasal administration of exogenous orexin A [36]. Interestingly, a human study showed that the levels of orexin $\mathrm{A}$ in the amygdala are maximal during positive emotion, social interaction, and anger [37]. On the other hand, the activation of orexin signaling during social defeat stress decreased subsequent social interaction and recognition memory in rats [38]. Totally, the data support an association between the orexin system and sociability behaviors.

The orexinergic neurons of the BLA express interactions with a number of neuronal populations involved in the regulation of social and emotional competence. The balance between excitatory and inhibitory transmission in the BLA is important for maintaining normal neuronal activity [39]. It is, therefore, likely that inactivation of OrxlR signaling in the BLA causes functional alterations in other receptor systems. Such an idea can be supported by the studies using genetic or pharmacological inhibition methodologies that demonstrate the interaction of OrxlR with various brain neurotransmitter systems including gamma 
aminobutyric acid, glutamate, and cannabinoid receptors [40-42]. However, challenges and open issues regarding the interplay between OrxlR and other receptor systems in the BLA need to be further investigated.

Specifically, our data indicated that intra-BLA OrxiR blockade had no significant effect on NTG-induced nociceptive hypersensitivity. There is no previous report that supports a possible role of OrxlR in the BLA in central sensory processing of pain signals. However, the association between sensory processing of migraine and OrxlR has been demonstrated in previous studies. Holland et al. [11], using a rat model with electrical stimulation of dura mater, showed that intravenous administration of orexin A suppressed trigeminal neuronal firing, and this effect was prevented by SB-334867, a selective Orx1R antagonist. Nonetheless, it has been indicated that chronic daily oral administration of filorexant, a dual orexin receptor antagonist, is not adequate for migraine prophylaxis. Such controversial results yielded by different studies may indicate the site-specific effects of OrxlR on the modulation of migraine pain.

The BLA has been shown to play a fundamental role in the processing of negative emotions and pain-related memories $[43,44]$. However, the involvement of the BLA in sensory input modulation is even more complicated by incorporating the $\mathrm{CeA}$ and prefrontal cortical circuitries $[43,45]$. Based on the findings in this study, it seems likely that the ability of OrxlR to modulate comorbid psychiatric disorders in patients with migraine is superior to its effect on sensory processing.

Accumulating evidence support the critical role of orexin system in the modulation of various types of pain including migraine [13]. In this study, unexpectedly, inactivation of OrxlR in the BLA did not change pain sensitivity (thermal) in the tail flick and hot plate test. None-the-less, SB-334867 administration could increase NTG-induced migraine-like spontaneous pain behavior. No change in thermal pain sensitivity can be somewhat related to methods used to measure hyperalgesia, SB-334867 doses, and the injection site in the BLA, which is a nonspecific brain structure for transmission and processing of nociceptive signals. In any case, additional studies are still required to determine the distinct roles of OrxlR within the BLA on pain sensitivity.

In this study, a limitation was that we evaluated the migraine model in only male rats. Based on epidemiological studies, there are sex-related differences in migraine phenotypes $[46,47]$. Neuroimaging studies in patients suffering from migraine have revealed anatomical and functional differences in regional brain activity between males and females [48,49]. In addition, puberty-related changes in syntheses and concentrations of sex hormones, mostly estrogen, play an important role in the frequency and severity of migraine headaches [47]. So, complementary research on females would be helpful in explaining the potential difference between the two sexes in vulnerability to NTG-induced migraine headache. Another limitation was that we evaluated NTG-related allodynia with only thermal stimuli. Allodynia in migraine is thermal, mechanical, dynamic, and mechanical static. Therefore, in this study, other components of allodynia were not evaluated.

Taken together, these findings suggest the potential value of orexinergic system of the BLA in the regulation of NTG-induced anxiety and social interaction deficits in rats. However, the blockade of OrxlR did not affect pain sensitivity, suggesting that OrxlR may play a more critical role in the modulation of comorbid psychiatric disorders rather than in sensory processing in patients with migraine.

\section{ACKNOWLEDGMENTS}

The authors wish to thank Shahid Bahonar University of Kerman and Kerman University of Medical Sciences for financial support.

\section{CONFLICT OF INTEREST}

No potential conflict of interest relevant to this article was reported.

\section{FUNDING}

This study was granted by Kerman University of Medical Sciences (grant number: 99-1).

\section{ORCID}

Khadijeh Askari-Zahabi, https://orcid.org/0000-0002-2242-0511

Mehdi Abbasnejad, https://orcid.org/0000-0003-2570-5288

Razieh Kooshki, https://orcid.org/0000-0001-6024-4507

Maryam Raoof, https://orcid.org/0000-0002-2628-2136

Saeed Esmaeili-Mahani, https://orcid.org/0000-0001-5550-9806

Ali Mohammad Pourrahimi, https://orcid.org/0000-0002-5495-5289

Mahnaz Zamyad, https://orcid.org/0000-0003-1716-5824

\section{REFERENCES}

1. Dodick DW. A phase-by-phase review of migraine patho- 
physiology. Headache 2018; 58 Suppl 1: 4-16.

2. Balaban CD, Jacob RG, Furman JM. Neurologic bases for comorbidity of balance disorders, anxiety disorders and migraine: neurotherapeutic implications. Expert Rev Neurother 2011; 11: 379-94.

3. Maizels M, Aurora S, Heinricher M. Beyond neurovascular: migraine as a dysfunctional neurolimbic pain network. Headache 2012; 52: 1553-65.

4. Hadjikhani N, Ward N, Boshyan J, Napadow V, Maeda Y, Truini A, et al. The missing link: enhanced functional connectivity between amygdala and visceroceptive cortex in migraine. Cephalalgia 2013; 33: 1264-8.

5. Chen Z, Chen X, Liu M, Dong Z, Ma L, Yu S. Altered functional connectivity of amygdala underlying the neuromechanism of migraine pathogenesis. J Headache Pain 2017; 18: 7.

6. Harrison DG, Bates JN. The nitrovasodilators. New ideas about old drugs. Circulation 1993; 87: 1461-7.

7. de Tommaso M, Libro G, Guido M, Difruscolo O, Losito L, Sardaro M, et al. Nitroglycerin induces migraine headache and central sensitization phenomena in patients with migraine without aura: a study of laser evoked potentials. Neurosci Lett 2004; 363: 272-5.

8. Kilduff TS, Peyron C. The hypocretin/orexin ligand-receptor system: implications for sleep and sleep disorders. Trends Neurosci 2000; 23: 359-65.

9. Flores Á, Herry C, Maldonado R, Berrendero F. Facilitation of contextual fear extinction by orexin-1 receptor antagonism is associated with the activation of specific amygdala cell subpopulations. Int J Neuropsychopharmacol 2017; 20: 6549.

10. Kim TK, Han PL. Functional connectivity of basolateral amygdala neurons carrying orexin receptors and melaninconcentrating hormone receptors in regulating sociability and mood-related behaviors. Exp Neurobiol 2016; 25: 307-17.

11. Holland PR, Akerman S, Goadsby PJ. Modulation of nociceptive dural input to the trigeminal nucleus caudalis via activation of the orexin 1 receptor in the rat. Eur J Neurosci 2006; 24: 2825-33.

12. Paxinos G, Watson C. Paxinos \& Watson the rat brain in stereotaxic coordinates. 6th ed. Amsterdam, Elsevier. 2007.

13. Kooshki R, Abbasnejad M, Esmaeili-Mahani S, Raoof M, Sheibani V. Activation orexin 1 receptors in the ventrolateral periaqueductal gray matter attenuate nitroglycerin-induced migraine attacks and calcitonin gene related peptide upregulation in trigeminal nucleus caudalis of rats. Neuropharmacology 2020; 178: 107981.

14. Raoof M, Shakoori A, Kooshki R, Abbasnejad M, Amanpour S. The effects of regular exercise on capsaicin-induced pulpal pain and pain-induced changes in passive avoidance learning and memory in rats. Korean J Pain 2017; 30: 258-64.

15. Yao G, Huang Q, Wang M, Yang CL, Liu CF, Yu TM. Behavioral study of a rat model of migraine induced by CGRP. Neu- rosci Lett 2017; 651: 134-9.

16. Huang D, Ren L, Qiu CS, Liu P, Peterson J, Yanagawa Y, et al. Characterization of a mouse model of headache. Pain 2016; 157: 1744-60.

17. Farkas S, Bölcskei K, Markovics A, Varga A, Kis-Varga Á, Kormos V, et al. Utility of different outcome measures for the nitroglycerin model of migraine in mice. J Pharmacol Toxicol Methods 2016; 77: 33-44

18. Bates EA, Nikai T, Brennan KC, Fu YH, Charles AC, Basbaum AI, et al. Sumatriptan alleviates nitroglycerin-induced mechanical and thermal allodynia in mice. Cephalalgia 2010; 30: 170-8.

19. Greco R, Demartini C, Zanaboni AM, Tassorelli C. Chronic and intermittent administration of systemic nitroglycerin in the rat induces an increase in the gene expression of CGRP in central areas: potential contribution to pain processing. J Headache Pain 2018; 19: 51.

20. Li Y, Zhang Q, Qi D, Zhang L, Yi L, Li Q, et al. Valproate ameliorates nitroglycerin-induced migraine in trigeminal nucleus caudalis in rats through inhibition of NF- $\kappa$ B. J Headache Pain 2016; 17: 49 .

21. Schwedt TJ. Multisensory integration in migraine. Curr Opin Neurol 2013; 26: 248-53.

22. Ashkenazi A, Silberstein S, Jakubowski M, Burstein R. Improved identification of allodynic migraine patients using a questionnaire. Cephalalgia 2007; 27: 325-9.

23. Bigal ME, Ashina S, Burstein R, Reed ML, Buse D, Serrano $\mathrm{D}$, et al. Prevalence and characteristics of allodynia in headache sufferers: a population study. Neurology 2008; 70: 152533.

24. Schwedt TJ, Krauss MJ, Frey K, Gereau RW 4th. Episodic and chronic migraineurs are hypersensitive to thermal stimuli between migraine attacks. Cephalalgia 2011; 31: 6-12.

25. Gottschalk MG, Richter J, Ziegler C, Schiele MA, Mann J, Geiger MJ, et al. Orexin in the anxiety spectrum: association of a HCRTR1 polymorphism with panic disorder/agoraphobia, CBT treatment response and fear-related intermediate phenotypes. Transl Psychiatry 2019; 9: 75.

26. Peres MFP, Vieira DS, Masruha MR, Gonçalves AL, Mercante JPP, Naffah-Mazzacoratti M da G. Orexin-A CSF levels correlate with anxiety but not excessive daytime sleepiness in chronic migraine. Headache Med 2011; 2: 41-5.

27. Johnson PL, Truitt W, Fitz SD, Minick PE, Dietrich A, Sanghani S, et al. A key role for orexin in panic anxiety. Nat Med 2010; 16: 111-5.

28. Flores Á, Valls-Comamala V, Costa G, Saravia R, Maldonado $\mathrm{R}$, Berrendero $\mathrm{F}$. The hypocretin/orexin system mediates the extinction of fear memories. Neuropsychopharmacology 2014; 39: 2732-41.

29. Wang C, Wang Q, Ji B, Pan Y, Xu C, Cheng B, et al. The orexin/ receptor system: molecular mechanism and therapeutic potential for neurological diseases. Front Mol Neurosci 2018; 
11: 220.

30. Schafe GE, Atkins CM, Swank MW, Bauer EP, Sweatt JD, LeDoux JE. Activation of ERK/MAP kinase in the amygdala is required for memory consolidation of pavlovian fear conditioning. J Neurosci 2000; 20: 8177-87.

31. Keil MF, Briassoulis G, Stratakis CA. The role of protein kinase A in anxiety behaviors. Neuroendocrinology 2016; 103: 625-39.

32. Yang L, Shi LJ, Yu J, Zhang YQ. Activation of protein kinase A in the amygdala modulates anxiety-like behaviors in social defeat exposed mice. Mol Brain 2016; 9: 3.

33. Di Benedetto B, Kallnik M, Weisenhorn DM, Falls WA, Wurst W, Hölter SM. Activation of ERK/MAPK in the lateral amygdala of the mouse is required for acquisition of a fear-potentiated startle response. Neuropsychopharmacology 2009; 34: 356-66.

34. Maldonado NM, Espejo PJ, Martijena ID, Molina VA. Activation of ERK2 in basolateral amygdala underlies the promoting influence of stress on fear memory and anxiety: influence of midazolam pretreatment. Eur Neuropsychopharmacol 2014; 24: 262-70.

35. Abbas MG, Shoji H, Soya S, Hondo M, Miyakawa T, Sakurai T. Comprehensive behavioral analysis of male Oxlr (-/-) mice showed implication of orexin receptor-1 in mood, anxiety, and social behavior. Front Behav Neurosci 2015; 9: 324.

36. Yang L, Zou B, Xiong X, Pascual C, Xie J, Malik A, et al. Hypocretin/orexin neurons contribute to hippocampusdependent social memory and synaptic plasticity in mice. J Neurosci 2013; 33: 5275-84.

37. Blouin AM, Fried I, Wilson CL, Staba RJ, Behnke EJ, Lam HA, et al. Human hypocretin and melanin-concentrating hormone levels are linked to emotion and social interaction. Nat Commun 2013; 4: 1547.

38. Eacret D, Grafe LA, Dobkin J, Gotter AL, Renger JJ, Winrow $\mathrm{CJ}$, et al. Orexin signaling during social defeat stress influences subsequent social interaction behaviour and recognition memory. Behav Brain Res 2019; 356: 444-52.

39. Wellman LL, Forcelli PA, Aguilar BL, Malkova L. Bidirection- al control of social behavior by activity within basolateral and central amygdala of primates. J Neurosci 2016; 36: 874656.

40. Flanigan ME, Aleyasin H, Li L, Burnett CJ, Chan KL, LeClair $\mathrm{KB}$, et al. Orexin signaling in GABAergic lateral habenula neurons modulates aggressive behavior. Nat Neurosci 2020; 23: 638-50.

41. Ward RJ, Pediani JD, Milligan G. Heteromultimerization of cannabinoid $\mathrm{CB}(1)$ receptor and orexin $\mathrm{OX}(1)$ receptor generates a unique complex in which both protomers are regulated by orexin A. J Biol Chem 2011; 286: 37414-28.

42. Aracri P, Banfi D, Pasini ME, Amadeo A, Becchetti A. Hypocretin (orexin) regulates glutamate input to fast-spiking interneurons in layer $\mathrm{V}$ of the $\mathrm{Fr} 2$ region of the murine prefrontal cortex. Cereb Cortex 2015; 25: 1330-47.

43. Li Z, Wang J, Chen L, Zhang M, Wan Y. Basolateral amygdala lesion inhibits the development of pain chronicity in neuropathic pain rats. PLoS One 2013; 8: e70921.

44. Cai YQ, Wang W, Paulucci-Holthauzen A, Pan ZZ. Brain circuits mediating opposing effects on emotion and pain. J Neurosci 2018; 38: 6340-9.

45. Kosarmadar N, Ghasemzadeh Z, Rezayof A. Inhibition of microglia in the basolateral amygdala enhanced morphineinduced antinociception: possible role of GABAA receptors. Eur J Pharmacol 2015; 765: 157-63.

46. Pavlovic JM, Akcali D, Bolay H, Bernstein C, Maleki N. Sexrelated influences in migraine. J Neurosci Res 2017; 95: 58793.

47. Delaruelle Z, Ivanova TA, Khan S, Negro A, Ornello R, Raffaelli B, et al. Male and female sex hormones in primary headaches. J Headache Pain 2018; 19: 117.

48. Maleki N. Gender differences in imaging studies in migraine. In: Gender and migraine. Edited by Maassen van den BrinkA, MacGregor EA. Cham, Springer. 2019, pp 101-11.

49. Maleki N, Barmettler G, Moulton EA, Scrivani S, Veggeberg R, Spierings ELH, et al. Female migraineurs show lack of insular thinning with age. Pain 2015; 156: 1232-9. 